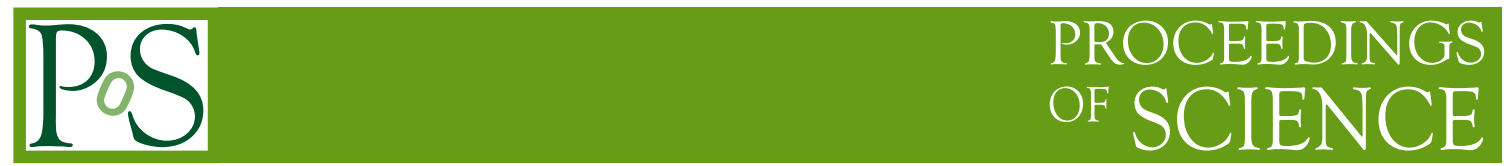

\title{
Non-Hermitian Polynomial Hybrid Monte Carlo
}

\section{Oliver Witzel*}

Humboldt Universität zu Berlin, Institut für Physik, Newtonstr. 15, 12489 Berlin, Germany

E-mail: witzel@physik.hu-berlin.de

We report on a new variant of the hybrid Monte Carlo algorithm employing a polynomial approximation of the inverse of the non-Hermitian Dirac-Wilson operator. Our approximation relies on simple and stable recurrence relations of complex Chebyshev polynomials. First performance figures are presented.

The XXVI International Symposium on Lattice Field Theory

July 14 - 19, 2008

Williamsburg, Virginia, USA

\footnotetext{
* Speaker.
} 


\section{Introduction}

Despite the steady progress in developing new machines providing more and more computational resources, two-flavor dynamical fermion simulations remain challenging. Moving on to large volume simulations [1] we experienced frequent occurrences of large energy violations within the hybrid Monte Carlo (HMC) update [2] indicating possible algorithmic instabilities and raising worries about reversibility violations. In [3] it is pointed out that such instabilities are caused by tiny eigenvalues of the Dirac-Wilson operator. Therefore we propose a new HMC variant reviving the idea to reweight observables and approximate the inverse, non-Hermitian Dirac-Wilson operator by Chebyshev polynomials. Our new variant is demonstrated using Wilson's lattice action [4]

$$
S\left(U_{\mu}, \bar{\psi}, \psi\right)=\frac{1}{g_{0}} \sum_{P} \operatorname{Tr}\left\{1-U_{P}\right\}+\sum_{x, y} \bar{\psi}(x) M_{x y} \psi(y),
$$

where the first sum runs over all plaquettes $U_{P}(x)=U_{\mu}(x) \cdot U_{v}(x+\hat{\mu}) \cdot U_{\mu}(x+\hat{v})^{\dagger} \cdot U_{v}(x)^{\dagger}$ of the gauge field $U_{\mu}(x)$ and the second sum over all lattice sites. $g_{0}$ is the strong coupling constant and $M$ is the non-Hermitian Dirac-Wilson operator given in matrix notation. After integrating out the quark fields (anti-commuting Grassmann variables) $\bar{\psi}$ and $\psi$ we arrive for the second summand at the fermion determinant $\operatorname{det}\{M\}$. Due to the sparse structure of $M$ it is suitable for even-odd preconditioning, which leads to a factorization of $\operatorname{det}\{M\}$ [5]

$$
\begin{aligned}
\operatorname{det}\{M\} & =\operatorname{det}\left\{\mathrm{M}_{\mathrm{ee}}\right\} \cdot \operatorname{det}\left\{\hat{M}^{A}\right\} \\
& =\operatorname{det}\left\{\mathrm{M}_{\mathrm{ee}}\right\} \cdot \operatorname{det}\left\{\hat{M}^{S}\right\} \cdot \operatorname{det}\left\{\mathrm{M}_{\mathrm{oo}}\right\} .
\end{aligned}
$$

The two possibilities are commonly named asymmetric and symmetric with the preconditioned operator $\hat{M}^{A}=\mathrm{M}_{\mathrm{oo}}-\mathrm{M}_{\mathrm{oe}} \mathrm{M}_{\mathrm{ee}}^{-1} \mathrm{M}_{\mathrm{eo}}$ and $\hat{M}^{S}=1-\mathrm{M}_{\mathrm{oo}}^{-1} \mathrm{M}_{\mathrm{oe}} \mathrm{M}_{\mathrm{ee}}^{-1} \mathrm{M}_{\mathrm{eo}}$, respectively.

Setting up an algorithm to simulate two flavor QCD, we consider the determinant of $M M^{\dagger}$. The algorithmic concept of our new HMC variant follows the concept of the polynomial hybrid Monte Carlo (PHMC) by Frezotti and Jansen [6]. They approximate the Hermitian operator by a root factorization [7] and allow to compensate by a reweighting factor for a possible deviation from importance sampling. Here we make use of the non-Hermitian, even-odd preconditioned operator $\hat{M}=\hat{M}^{S}$ or $\hat{M}^{A}$, introduce polynomials $P_{n} \approx \hat{M}^{-1}$ and incorporate as well a reweighting factor

$$
\operatorname{det}\left\{\hat{M} \hat{M}^{\dagger}\right\}=\operatorname{det}\left\{\left[\hat{M} P_{n}\right]\left[\hat{M} P_{n}\right]^{\dagger}\right\} \cdot\left[\operatorname{det}\left\{P_{n} P_{n}^{\dagger}\right\}\right]^{-1} .
$$

In the next section we first motivate our choice of the non-Hermitian Dirac-Wilson operator and show some of its properties. Our approximation of the inverse Dirac-Wilson operator in terms of Chebyshev polynomials is presented in Section 3 and in Section 4 we introduce the basic steps of our Non-Hermitian Polynomial Hybrid Monte Carlo (NPHMC). The dependence on the polynomial parameters is analyzed in Section 5.

\section{Non-Hermitian Dirac-Wilson Operator}

Using matrix notation and the hopping parameter representation we can write the Dirac-Wilson operator as $M_{x y}=\delta_{x y}-K_{x y}$, where all interactions are contained in

$$
K_{x y}=\kappa\left(H_{x y}-\frac{i}{2} c_{\mathrm{sw}} \sigma_{\mu \nu} \mathscr{F}_{\mu \nu} \delta_{x y}\right) .
$$


$K_{x y}$ is thus built up by the hopping operator $H_{x y}$ and the $O(a)$ improvement, the SheikholeslamiWohlert-term, and is proportional to the hopping parameter $\kappa$. In [8] we present spectral properties of this operator in a set-up with Schrödinger functional (SF) boundary conditions (BC). For our further considerations the following properties are important:

- $M$ has a complex spectrum lying in the positive half-plane bounded by an ellipse

- $M^{-1}$ can be approximated recursively by simple and stable polynomials

- We expect a "good" approximation with a lower degree polynomial than if approximating the Hermitian operator, which is indicated by a lower condition number ${ }^{1}$

- These properties carry over to the even-odd preconditioned operator $\hat{M}$

The latter manifests itself in an exact relation between the eigenvalues $\hat{\lambda}$ of the preconditioned operator $\hat{K}$ and the eigenvalues $\lambda$ of $K$ if the $O(a)$ improvement is switched off $\left(c_{\mathrm{sw}}=0\right)$

$$
\hat{\lambda}(\hat{K})=\lambda^{2}(K) .
$$

Assuming next the spectrum of $K$ and $\hat{K}$, respectively, to fill perfectly an ellipse we parameterize the eigenvalues of $K$ by $\lambda=e \cosh (\vartheta+i \phi)$ with eccentricity $e$ and "angles" $\vartheta$ and $\phi$. From (2.2) follows now for the spectrum of the preconditioned operator

$$
\hat{e}=\hat{\delta}=e^{2} / 2,
$$

where $\hat{e}$ is the eccentricity of the ellipse bounding the spectrum of $\hat{K}$ and $\hat{\delta}$ marks a positive shift along the real axis. With $O(a)$ improvement turned on, (2.2) as well as the drawn conclusions hold only approximately.

Computing the spectral boundary numerically by using the complex Lanczos method we can nicely visualize the shift and the advantage due to even-odd preconditioning (see Fig. 1). Moreover we see that with Sheikholeslami-Wohlert term the symmetric version of even-odd preconditioning is superior because it leads to a more compact and round spectrum. In addition one can verify certain symmetries of the operator (see e.g. [9]): without the clover term and if all dimensions of the lattice are even, the spectrum exhibits a mirror symmetry under sign flip and has complex pairs of eigenvalues ( $\gamma_{5}$ Hermiticity). With clover term the first is not present, but we still find complex pairs of eigenvalues.

\section{Chebyshev Approximation}

In order to find a polynomial approximation of the inverse, non-Hermitian Dirac-Wilson operator $\hat{M}$ we first introduce a "small quantity" (remainder)

$$
R_{n+1}(\hat{M})=\mathbb{1}-\hat{M} P_{n} .
$$

By construction $R_{n+1}$ is small on an elliptical region of the spectrum of $\hat{M}$ and suitable for an approximation by scaled and translated Chebyshev polynomials as introduced by Manteuffel [10]

$$
R_{n+1}(\hat{M})=\frac{T_{n+1}(\hat{K} / \hat{e})}{T_{n+1}(\hat{d} / \hat{e})} .
$$

\footnotetext{
${ }^{1}$ We thank Tony Kennedy for pointing that out.
} 

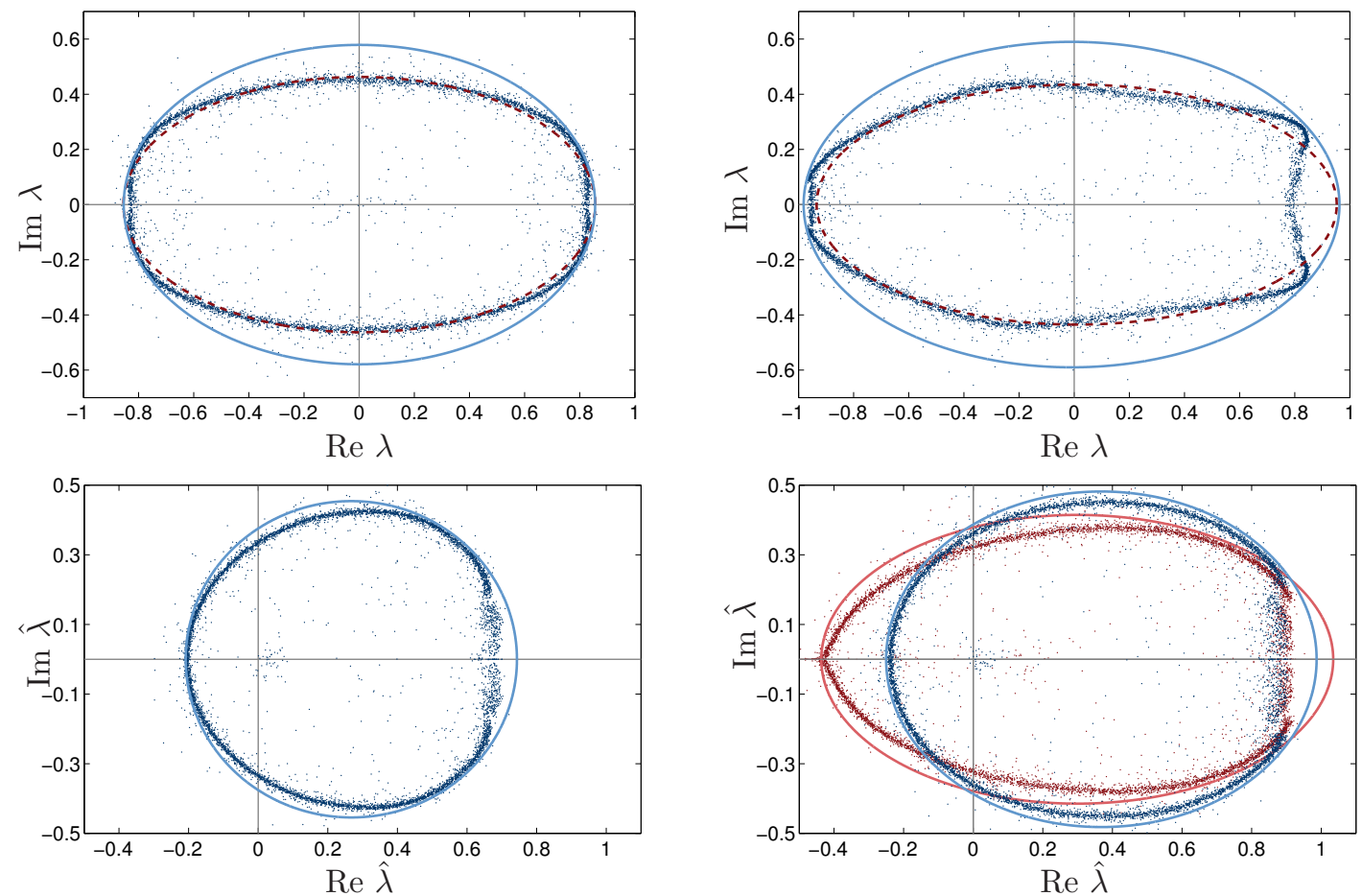

Figure 1: Computing the spectral boundary of $1-M$ (upper plots) and $\mathbb{1}-\hat{M}$ (lower plots) by the Lanczos method on a set of 50 pure-gauge configurations: $8^{4}$ lattice at $\beta=6.0$ and $\kappa=0.13458$ in the SF. Left without / right with clover-term; lower right plot shows in red asymmetric and in blue symmetric even-odd preconditioning.

Here $\hat{d}=1+\hat{\delta}$ is ideally such that the spectrum of $\hat{K}$ is origin centered and hence $\hat{M}=\hat{d}-\hat{K}$. The spectral region approximated is bounded by an ellipse with eccentricity $\hat{e}$. Eq. (3.2) together with the well-known recurrence relation for Chebyshev polynomials,

$$
T_{n+1}(z)=2 z T_{n}(z)-T_{n-1}(z) \quad \text { with } \quad T_{1}=z \quad \text { and } \quad T_{0}=1 \text {, }
$$

provides the key to obtain first a recursive description for the $R_{n+1}$ and by (3.1) also for our sought polynomials $P_{n}$ [11],

$$
\begin{aligned}
& R_{n+1}=a_{n} \hat{K} R_{n}+\left(1-\hat{d} a_{n}\right) R_{n-1} \quad \text { with } \quad R_{1}=\hat{K} / \hat{d} \quad \text { and } \quad R_{0}=1 \text {, } \\
& P_{n}=a_{n}\left(1+\hat{K} P_{n-1}\right)+\left(1-\hat{d} a_{n}\right) P_{n-2} \quad \text { with } \quad P_{1}=a_{1}(1+\hat{K} / \hat{d}) \quad \text { and } \quad P_{0}=\mathbb{1} / \hat{d} \text {, }
\end{aligned}
$$

The real coefficients $a_{n}$ are given by

$$
a_{n}=\left(\hat{d}-a_{n-1} \hat{e}^{2} / 4\right)^{-1} \quad \text { with } \quad a_{1}=\hat{d}\left(\hat{d}^{2}-\hat{e}^{2} / 2\right)^{-1} .
$$

and converge to $\lim _{n \rightarrow \infty} a_{n}=2\left(\hat{d}-\hat{e} \sqrt{\hat{d}^{2} / \hat{e}^{2}-1}\right) / \hat{e}^{2}$. Concerning the two recurrence relations (3.4) and (3.5) we like to emphasize: although given as matrix relations, (3.4) and (3.5) lead to repeated, numerically cheap matrix times vector multiplications. Furthermore, the inverting polynomial is obtained from numerically stable and simple two-step recursions.[12] 


\section{NPHMC update}

To generate configurations with the Boltzmann weight $\exp \{-S\}$ by a NPHMC update, we manipulate the action (1.1) accordingly

$$
S=\frac{1}{g_{0}} \sum_{P} \operatorname{Tr}\left\{1-U_{P}\right\}+2\left[\ln \operatorname{det}\left\{\mathrm{M}_{\mathrm{ee}}\right\}+\ln \operatorname{det}\left\{\mathrm{M}_{\mathrm{oo}}\right\}\right]+\underbrace{\phi^{\dagger} P_{n}^{\dagger}(\hat{M}) P_{n}(\hat{M}) \phi}_{S_{b}},
$$

where from now on we choose $\hat{M}=\hat{M}^{S}$ since $\hat{M}^{S}$ is superior to $\hat{M}^{A}$. The first term is the unchanged contribution from the gauge fields, it follows the determinant contribution due to even-odd preconditioning and the last term gives rise to the bosonic contribution obtained by estimating the second factor of the right hand side in (1.4) as bosonic integral. We take care of the first factor in (1.4), $\operatorname{det}\left\{\left[\hat{M} P_{n}\right]\left[\hat{M} P_{n}\right]^{\dagger}\right\}$, by calculating a stochastic estimate using random Gaussian fields $\eta_{C}$

$$
C=\exp \left\{\eta_{C}^{\dagger}\left[1-\left(P_{n}^{\dagger} \hat{M}^{\dagger} \hat{M} P_{n}\right)^{-1}\right] \eta_{C}\right\} .
$$

This term is only computed when measuring observables. The general procedure of an HMC update is unchanged. Differences occur in the way the bosonic contribution $S_{b}$ is treated.

At the beginning of a trajectory the pseudo-fermion fields $\phi$ have to be generated with the correct distribution by inverting $P_{n}$ on a random Gaussian field $\eta$

$$
\phi=P_{n}^{-1} \eta=\left(1-R_{n+1}\right)^{-1} \hat{M} \eta .
$$

Here it is important to use the second relation and by that invert a well conditioned matrix needing only a little number of expensive iteration steps. Next we compute the variation $\delta\left\{S_{b}\right\}$ as it is required to integrate the equations of motions. Varying each occurrence of $\hat{K}$ and reorganizing the expressions we yield

$$
\delta\left\{S_{b}\right\}=\sum_{l=1}^{n}\left[\xi_{2 n-l}^{\dagger} a_{l} \delta\{\hat{K}\} \chi_{l-1}+\text { H.c. }\right]
$$

with

$$
\begin{aligned}
& \chi_{j}=a_{j} \phi+a_{j} \hat{K} \chi_{j-1}+\left(1-\hat{d} a_{j}\right) \chi_{j-2} ; \quad \chi_{1}=a_{1}(1+\hat{K} / \hat{d}) \phi ; \quad \chi_{0}=\phi / d \\
& \xi_{n+j}^{\dagger}=\xi_{n+j-1}^{\dagger} \hat{K} a_{n-j+1}+\xi_{n+j-2}^{\dagger}\left(1-\hat{d} a_{n-j+2}\right) ; \quad \xi_{n+1}^{\dagger}=\chi_{n}^{\dagger} \hat{K} a_{n} ; \quad \xi_{n}^{\dagger}=\chi_{n}^{\dagger} \text {. }
\end{aligned}
$$

Finally, the reweighting factor $C$ is estimated requiring again the inversion of a well-conditioned matrix. Of course also here we can replace $\hat{M} P_{n}$ by $11-R_{n+1}$. For further details see [12].

\section{Performance Tests}

Testing the proposed new HMC variant we investigate the dependence on the three polynomial parameters $\hat{\delta}, \hat{e}$ and $n$ on an $8^{4}$ lattice at $\beta=6.0$ and $\kappa=0.13458$. The tests are performed by keeping two parameters fixed, while varying the third one and monitoring as observables: the mean value of the correction factor $\langle C\rangle$, the relative width of its distribution $\varsigma_{C}=\sqrt{\left\langle C^{2}\right\rangle-\langle C\rangle^{2}} /\langle C\rangle$ as 

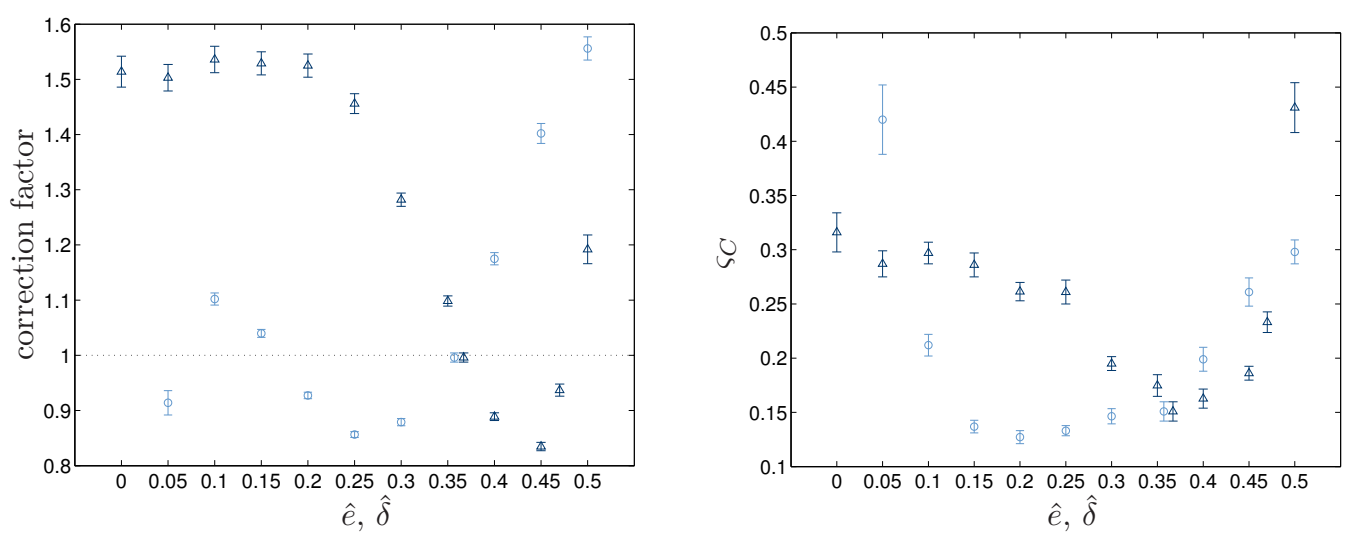

Figure 2: Dependence of the correction factor $C$ and the width of its distribution $\varsigma_{C}$ on $\hat{\delta}$ (light blue $\circ$ ) and $\hat{e}($ dark blue $\triangle$ ), respectively.

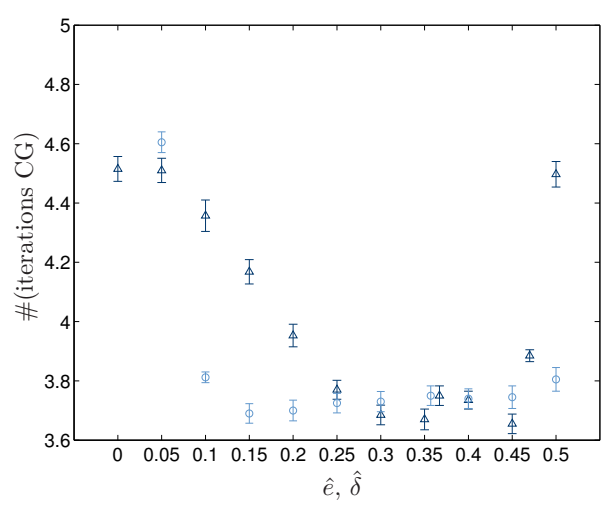

Figure 3: Dependence of the number of CF iterations on $\hat{\delta}$ (light blue $\circ$ ) and $\hat{e}$ (dark blue $\triangle$ ), respectively.

well as the number of conjugate gradient (CG) iterations required to compute the correction factor, \#(iterations CG).

As can be seen by looking at Figs. 2 and 3 the two parameters specifying the elliptical region of the approximating polynomial favor $\hat{e} \approx \hat{\delta} \approx 0.36$ but do not require a sophisticated fine tuning. This value is in good agreement with the ones concluded from the bounding ellipse shown in Fig. 1 in case of symmetric even-odd preconditioning.

Turning to the dependence on the polynomial degree $n$ (Fig. 4) we find a much stronger dependence. This parameter is mainly responsible for the quality of our approximation. Hence it affects the cost as well as the noisiness of the correction factor. For a higher degree polynomial $C$ approaches one, while $\varsigma_{C}$ and \#(iterations CG) go to zero. Using the $4 n \cdot \#($ iterations CG) as a coarse estimate for the cost, a good choice of the polynomial degree - for this set-up - is $n=50$.

\section{Conclusion}

Employing the scaled and translated Chebyshev polynomials to approximate the inverse, nonHermitian Dirac-Wilson operators allows to derive a variant of the HMC update algorithm based on simple and stable recurrence relations. The approximation does not require a fine tuning of the parameters specifying the elliptical approximation region, whereas the polynomial degree $n$ is important for the quality of the approximation and the numerical cost. 


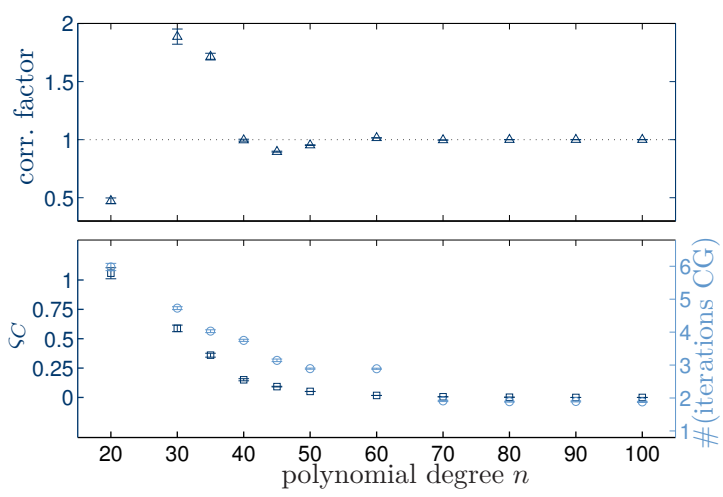

Figure 4: Dependence of $C(\triangle), \varsigma_{C}(\square)$ and \#(iterations CG) (०) on the polynomial degree $n$.

A conclusive comparison between the performance of different HMC-type algorithm has not been performed yet. However, the first data indicate that the 1-pseudo-fermion NPHMC is slightly superior than a standard 1-pseudo-fermion HMC, but inferior to an HMC version incorporating the Hasenbusch-trick [13] and multiple time scale integration [14]. Constructing a NPHMC with two pseudo-fermions by means of the Hasenbusch-trick is possible. Unfortunately, this forces an involved tuning of the polynomial degrees and appears to be not too promising.

As a byproduct of our studies of the non-Hermitian Dirac-Wilson operator, we found that the symmetric even-odd preconditioned operator is advantageous because of a more compact spectrum.

\section{Acknowledgement}

The author thanks B. Bunk and U. Wolff for fruitful discussions and the APE team at DESY Zeuthen for technical support. This work is supported by the DFG within the SFB/TR 9.

\section{References}

[1] M. Della Morte et al., PoS LAT2007 (2007) 255.

[2] S. Duane, A. D. Kennedy, B. J. Pendleton and D. Roweth, Phys. Lett. B195 (1987) 216.

[3] L. Del Debbio et al., JHEP 02 (2006) 011; M. Della Morte et al., JHEP 07 (2008) 037.

[4] K. G. Wilson, Phys. Rev. D10 (1974) 2445.

[5] K. Jansen and C. Liu, Comput. Phys. Commun. 99 (1997) 221.

[6] R. Frezzotti and K. Jansen, Phys. Lett. B402 (1997) 328.

[7] Ph. de Forcrand and T. Takaishi, Nucl. Phys. Proc. Suppl. 53 (1997) 968.

[8] S. Takeda, O. Witzel and U. Wolff, PoS LAT2007 (2007) 046.

[9] Ch. Gattringer and I. Hip, Nucl. Phys. B536 (1998) 363.

[10] Th. A. Manteuffel, Numer. Math. 28 (1977) 307.

[11] B. Bunk, Chebyshev Polynomials (1997) Internal Notes; U. Wolff, NPHMC (2001) Internal Notes.

[12] O. Witzel, Ph.D. Thesis Humboldt Universtität zu Berlin, 2008, to appear: http://edoc.hu-berlin.de/.

[13] M. Hasenbusch, Phys. Lett. B519 (2001) 177.

[14] C. Urbach et al., Comput. Phys. Comm. 174 (2006) 87. 